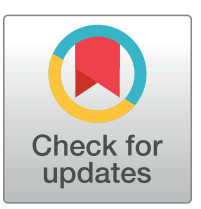

\section{G OPEN ACCESS}

Citation: Aguilera C, Gabau E, Ramirez-Mallafré A, Brun-Gasca C, Dominguez-Carral J, Delgadillo V, et al. (2021) New genes involved in Angelman syndrome-like: Expanding the genetic spectrum. PLoS ONE 16(10): e0258766. https://doi.org/ 10.1371/journal.pone.0258766

Editor: Klaus Brusgaard, Odense University Hospital, DENMARK

Received: November 10, 2020

Accepted: October 6, 2021

Published: October 15, 2021

Copyright: ๑ 2021 Aguilera et al. This is an open access article distributed under the terms of the Creative Commons Attribution License, which permits unrestricted use, distribution, and reproduction in any medium, provided the original author and source are credited.

Data Availability Statement: All relevant data are within the paper and its Supporting Information files.

Funding: This work is supported by Instituto de Salud Carlos III (MG, PI16/01411), Asociación Española de Síndrome de Angelman (EG), Institut d'investigació i innovació Parc Taulí I3PT (CA, CIR2016/025, CIR2018/021) and Ministerio de Economía y Competitividad (XD, SAF2016-14 $80255-\mathrm{R})$. The funders had no role in study design,

RESEARCH ARTICLE

\title{
New genes involved in Angelman syndrome- like: Expanding the genetic spectrum
}

\author{
Cinthia Aguilera ${ }^{1}$, Elisabeth Gabau ${ }^{2}$, Ariadna Ramirez-Mallafré ${ }^{2}$, Carme Brun-Gasca ${ }^{2,3}$, \\ Jana Dominguez-Carral ${ }^{2}$, Veronica Delgadillo ${ }^{2}$, Steve Laurie ${ }^{4}$, Sophia Derdak ${ }^{4}$, \\ Natàlia Padilla ${ }^{5}$, Xavier de la Cruz ${ }^{5,6}$, Núria Capdevila ${ }^{2}$, Nino Spataro ${ }^{1}$, Neus Baena ${ }^{1}$, \\ Miriam Guitart ${ }^{1 *}$, Anna Ruiz ${ }^{1 *}$
}

1 Genetics Laboratory, UDIAT-Centre Diagnòstic, Parc Taulí Hospital Universitari, Institut d'Investigació i Innovació Parc Taulí I3PT, Universitat Autònoma de Barcelona, Sabadell, Spain, 2 Paediatric Unit, Parc Taulí Hospital Universitari, Institut d'Investigació i Innovació Parc Taulí I3PT, Universitat Autònoma de Barcelona, Sabadell, Spain, 3 Department of Clinical Psychology and Health Psychology, Universitat Autònoma de Barcelona, Bellatera, Barcelona, Spain, 4 CNAG-CRG, Centre for Genomic Regulation (CRG), The Barcelona Institute of Science and Technology, Barcelona, Spain, 5 Neurosciences Area, Vall d'Hebron Institute of Research (VHIR), Universitat Autònoma de Barcelona, Barcelona, Spain, 6 Institució Catalana de Recerca i Estudis Avançats (ICREA), Barcelona, Spain

*aruizn@tauli.cat (AR); mguitart@tauli.cat (MG)

\section{Abstract}

Angelman syndrome (AS) is a neurogenetic disorder characterized by severe developmental delay with absence of speech, happy disposition, frequent laughter, hyperactivity, stereotypies, ataxia and seizures with specific EEG abnormalities. There is a $10-15 \%$ of patients with an AS phenotype whose genetic cause remains unknown (Angelman-like syndrome, AS-like). Whole-exome sequencing (WES) was performed on a cohort of 14 patients with clinical features of AS and no molecular diagnosis. As a result, we identified 10 de novo and $1 \mathrm{X}$-linked pathogenic/likely pathogenic variants in 10 neurodevelopmental genes ( $S Y N$ GAP1, VAMP2, TBL1XR1, ASXL3, SATB2, SMARCE1, SPTAN1, KCNQ3, SLC6A1 and $\angle A S 1 L)$ and one deleterious de novo variant in a candidate gene (HSF2). Our results highlight the wide genetic heterogeneity in AS-like patients and expands the differential diagnosis.

\section{Introduction}

Angelman syndrome (AS, OMIM \#105830) is a neurogenetic disorder with a prevalence of about $1 / 15000$ births. AS is characterized by severe developmental delay/intellectual disability (DD/ID) with absence of speech and distinctive dysmorphic craniofacial features such as microcephaly and wide mouth. Neurological problems include ataxia and seizures with specific electroencephalogram (EEG) abnormalities. The behavioral phenotype is characterized by happy disposition, frequent laughter, hyperactivity and stereotypies [1]. The consensus criteria for the clinical diagnosis of AS was proposed in 2005 by Williams et al., [1] which included a list of (i) consistent, (ii) frequent and (iii) associated features. However, clinical manifestations of AS can overlap with other diseases. 
data collection and analysis, decision to publish, or preparation of the manuscript.

Competing interests: The authors have declared that no competing interests exist.
AS is caused by the loss of function in neuronal cells of the ubiquitin protein ligase E6-AP (E6-Associated Protein) encoded by the UBE3A gene, which is located on chromosome 15q11-q13 imprinted region. Methylation study of this region identifies $75-80 \%$ of AS patients including maternal deletion, paternal uniparental disomy (UPD) and imprinting center defects. Pathogenic or likely pathogenic variants in the UBE3A gene identify a further $10 \%$ of cases. However, for approximately $10-15 \%$ of clinically diagnosed AS patients, the genetic cause remains unknown (AS-like) [2].

Some of these AS-like patients present alternative clinical and molecular diagnoses in syndromes that have overlapping clinical phenotypes and that should be considered in the differential diagnosis of AS. AS differential diagnosis include single gene disorders such as Christianson syndrome (SLC9A6), Rett syndrome (MECP2), Pitt Hopkins syndrome (TCF4), Kleefstra syndrome (EHMT1), Mowat-Wilson syndrome (ZEB2) or HERC2 deficiency syndrome (HERC2). Individuals affected by the above mentioned syndromes present severe DD, seizures, postnatal microcephaly, absent or minimal speech and sleep disturbances as AS patients [3-5].

In order to further identify the molecular defects in AS-like patients, whole exome sequencing (WES) was performed in a cohort of 13 parent-patient trios and one single patient with clinical features of AS and no molecular diagnosis. Pathogenic/likely pathogenic variants in known neurodevelopmental genes were found in $78,5 \%$ of patients while a deleterious variant in a new candidate gene was identified in another patient. Overall, our results show that 10 $15 \%$ of patients with a clinical but with no molecular diagnosis of AS present alternative genetic alterations in genes not previously associated with AS, expanding the genetic spectrum of AS-like.

\section{Material and methods}

\section{Patient samples}

14 patients ( 7 girls and 7 boys) referred to the Angelman syndrome Unit at the Parc Tauli Hospital Universitari (Sabadell, Spain) were enrolled in the study. Patient 1 has also been included in another study [6]. The corresponding written informed consent was obtained from all parents of each participant. The study was approved by the institutional Ethics Committee of Institut d'Investigació i Innovació Parc Taulí I3PT (CEIC 2016/668).

The clinical diagnosis was made between ages 11 months and 8 years. All patients presented neurodevelopmental phenotypes suggestive of AS including severe global developmental delay, speech impairment and a behavioral phenotype that included apparent happy disposition as the most remarkable feature. AS negative testing included the analysis of the methylation status of the SNURF-SNRPN locus and Sanger sequencing and intragenic deletions/ duplications analysis of the UBE3A gene. In addition, no alterations were detected by array comparative genomic hybridization (aCGH, ISCA $60 \mathrm{~Kb}$, Agilent Technologies) and fragile X syndrome testing.

All the cases were sporadic and no other relevant findings were present in their family history.

\section{Whole-exome sequencing and variant interpretation}

Trio WES of 13 patients and their parents was performed using the SureSelect Human All Exon V5+UTR kit (Agilent technologies). In patient 4, WES was performed only in the patient sample. Sequencing was performed on an Illumina Hiseq2000 platform (Illumina, San Diego, CA, USA) producing 2x100nt paired end reads at the National Centre of Genomic Analysis (CNAG-CRG, Barcelona, Spain). Raw data quality was assessed using FastQC software 
(v0.11.8, available at https://www.bioinformatics.babraham.ac.uk/projects/fastqc/) and an in depth analysis of each single generated FastQ file was performed to discard sequencing systematic errors and biases. On average, approximately 102.4 million reads per sample were generated during the sequencing process with an average GC content of $47.4 \%$ (standard deviation $=0.6 \%$ ). Each sequenced base had on average a coverage of $67 \mathrm{x}$ and for each individual $87 \%$ of bases had a coverage $>15$. Raw reads were mapped to the human reference genome (hg19) using the Burrows-Wheeler aligner (BWA, v0.7.17-r1188) [7] and subsequently processed using the Genome Analysis Toolkit (GATK) pipeline in order to remove PCR duplicates and perform base quality score recalibration. Reads with RMS Mapping Quality (MQ) = 255, with bad mates or a Phred mapping quality $<20$ were filtered out, only bases with Phred quality score $>18$ were considered for variant calling and only variants with Phred-scaled confidence $>10$ were called. Variant discovery was performed using the Haplotype Caller tool and following the best practices for exome sequencing variant discovery of GATK (v4.0.11.0) [8]. On average, 21,170 exonic variants were detected for each individual, among which on average 9,570 were missense variants, 351 were loss of functions, 237 were non-frameshift variants and 10,606 were synonymous variants. The remaining exonic variants were classified as "unknown" by ANNOVAR.

All exome variants were first checked against a de novo followed by an X-linked and autosomal recessive model of inheritance. In order to detect de novo variants, only variants with valid genotype and genotype quality $\geq 20$ in all the trio members were considered. Variants having a read depth lower than 5 in the parents or lower than 10 in the patients were discarded. Only variants that were heterozygous in the patients but homozygous for the reference allele in the parents were considered. Finally, putative de novo variants were filtered considering only those showing the alternative allele in more than $10 \%$ of the reads.

According to an autosomal recessive model of inheritance, annotated variants were filtered for allele frequencies $<0.02$ in the gnomAD database (v2.1.1) and their predicted impact on the protein. X-linked variants were filtered for allele frequencies $<0.001$ and their predicted impact on the protein.

Both de novo and recessive variants were annotated using ANNOVAR (v:16.04.2018) [9] a tool suited for functional annotation of variants detected from high-throughput sequencing data and assessing the impact of missense variants leveraging several in silico tools (S1 Table). Splice site variants were evaluated using the software Human Splicing Finder [10].

Sanger sequencing of the candidate variants was performed in the patients and the parents in order to confirm the presence of the variant and the pattern of inheritance. Variants were classified following the American College of Medical Genetics and Genomics and the Association for Molecular Pathology (ACMG/AMP) guidelines [11]. Pathogenic and likely pathogenic variants have been submitted to ClinVar [12].

\section{Real time quantitative PCR (RTqPCR) analysis}

RNA was extracted using the Biostic Blood Total RNA Isolation Kit sample (MO BIO laboratories, Inc) and cDNA was obtained using the PrimeScript ${ }^{\mathrm{TM}} \mathrm{RT}$ reagent Kit (Takara). RTqPCR gene expression analysis was performed in triplicate using the Taqman probes HSF2-Hs00988309_g1 and GADPH-Hs02758991_g1 for normalization (Applied Biosystems).

\section{Results}

Identified variants were first filtered according to a dominant de novo model of inheritance. Variants in genes known to be involved in neurodevelopmental diseases were prioritized and confirmed to be de novo. Overall, 10 de novo (SYNGAP1, VAMP2, TBL1XR1, ASXL3, SATB2, 
SMARCE1, SPTAN1, KCNQ3 and SLC6A1) and $1 \mathrm{X}$-linked (LAS1L) pathogenic and likely pathogenic variants were confirmed in 11 patients, leading to a diagnostic yield of $78,5 \%$ (Table 1). These variants were located in 10 different genes previously reported to be associated with neurodevelopmental disorders [6,13-21].

Clinical re-evaluation of patients at the time of the molecular diagnosis (ages between 9-38 years) showed that all patients met the consistent clinical features of AS (Table 2), except for ataxia of gait which was present in 9 of 14 patients. Even though the ataxia of gait is considered a consistent feature in AS patients, a recent review shows that it ranges from $72,7 \%$ to $100 \%$ according to the genetic etiology [27]. Additional clinical features identified in the clinical reevaluation of patients were analyzed taking into account the clinical phenotype described for the genes identified. The presence of specific clinical features associated with the new genes were confirmed for some of the patients. In short, cerebellar atrophy for SPTAN1 [13], hypoplasia of the corpus callosum, hypoplasia of the $5^{\text {th }}$ finger nail, hypertrichosis, sparse scalp hair and aggressive behavior for SMARCE1 [21], truncal obesity and short stature for LAS1L [16], myoclonic atonic seizures for SLC6A1 [15], aggressive behavior for SYNGAP1 [17], dysmorphic features and dental anomalies for ASXL3 [14] and aggressive behavior and dental anomalies for SATB2 [19] (Fig 1).

However, not all patients presented all the clinical features associated with the genes identified. Unsteady gait and hypotonia were not present in patient carrying the pathogenic variant in SYNGAP1 [17]. The patient harboring a pathogenic variant in SATB2 did not show sialorrhea and feeding difficulties [19]. Finally, the ataxia of gait, stereotypies and hypotonia were not observed in the patient with a pathogenic variant in KCNQ3 [20].

A novel candidate variant was identified in a gene not previously associated with neurodevelopmental disorders. The identified variant is a de novo frameshift deletion c.456_459delTGAG (NM_004506.3), p.(Ser152Argfs*40) in HSF2 gene in patient 12. The variant has not been reported before and is not present in the gnomAD database (version 2.1.1).

UBE3A has been shown to have both nuclear and cellular functions mainly through its ubiquitin protein-ligase activity [28]. UBE3A interacts with most of the components of the proteasome [29] regulating the activity of signal transduction pathways such as Wnt signaling that regulates central nervous system development [30-32] and synaptic plasticity in both excitatory and inhibitory GABAergic axon terminals [33-36]. At the nucleus, UBE3A has been shown to regulate chromatin structure, DNA methylation and transcriptional regulation [3740]. Interestingly, 8 out of the 10 genes found mutated in this study are mainly involved in synapsis (VAMP2, SYNGAP1, SLC6A1 and KCNQ3) [41-44] and chromatin remodeling or transcription regulation (TBL1XR1, SATB2, SMARCE1 and ASXL3) [45-48].

\section{Discussion}

We identified causal variants in 11 out of 14 patients with an AS-like phenotype. The global yield diagnostic of WES in this is study is $78,5 \%$, which is higher to what has been reported in the literature for other neurodevelopmental disorders (24-68\%) [49]. The results of WES led to the identification of 10 new genes that cause an AS-like phenotype (SYNGAP1, VAMP2, TBL1XR1, ASXL3, SATB2, SMARCE1, SPTAN1, KCNQ3, SLC6A1 and LAS1L), all of them previously associated with other neurodevelopmental disorders. In addition, we propose HSF2 (Heat Shock Factor) as a new candidate gene for the AS-like phenotype. Although HSF2 has not been previously associated with any human disease, the gene is highly expressed in the brain (Data source: GTEx Analysis Release V8, dbGaP Accession phs000424.v8.p2 [50]) and is highly intolerant to loss of function variation (pLI 0.92). Quantification of the mutated allele in mRNA showed a reduction in the allele carrying the frameshift variant (S1A Fig), suggesting 


\begin{tabular}{|c|c|c|c|c|c|c|c|c|c|c|c|}
\hline 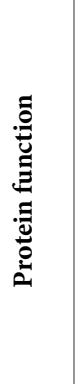 & 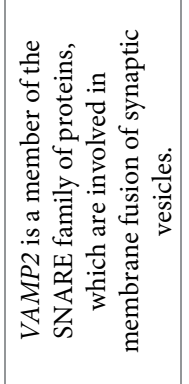 & 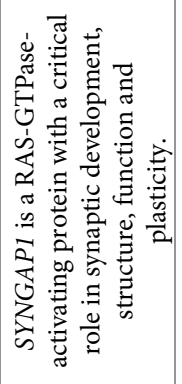 & 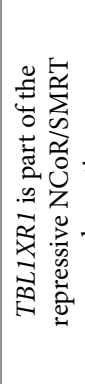 & 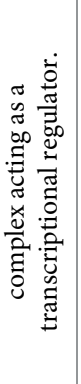 & 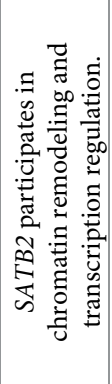 & 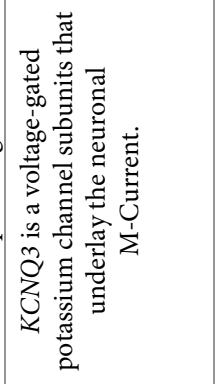 & 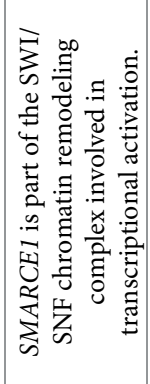 & 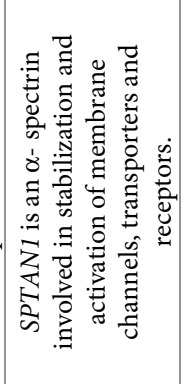 & 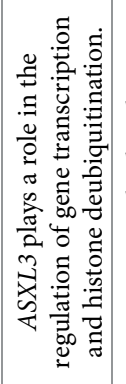 & 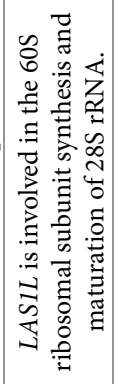 & 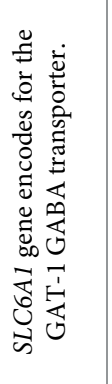 \\
\hline 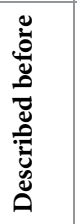 & 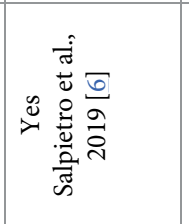 & $\stackrel{\circ}{z}$ & $\stackrel{\circ}{z}$ & $\stackrel{\circ}{z}$ & $\stackrel{\circ}{z}$ & 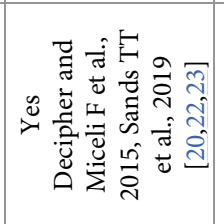 & 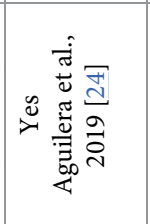 & $\stackrel{\circ}{z}$ & 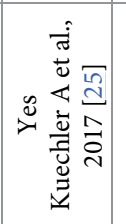 & ż & 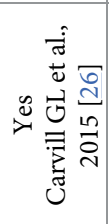 \\
\hline 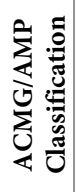 & 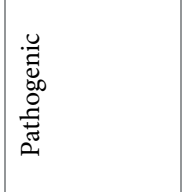 & 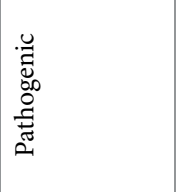 & 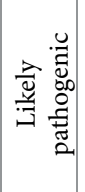 & 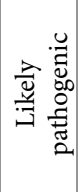 & 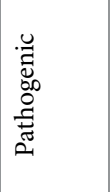 & 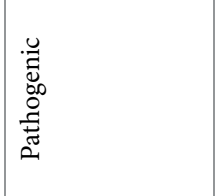 & 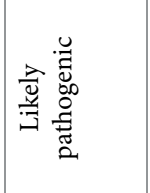 & 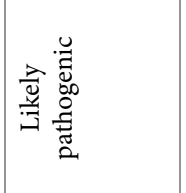 & 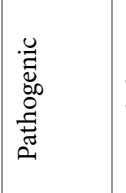 & 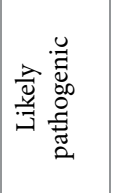 & 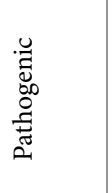 \\
\hline 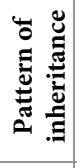 & 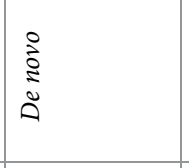 & 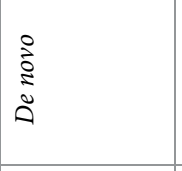 & 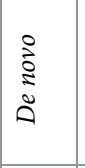 & 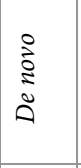 & 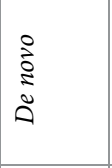 & $\begin{array}{l}\text { oे } \\
\text { ڤे } \\
\text { م̃ }\end{array}$ & 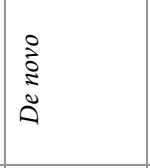 & 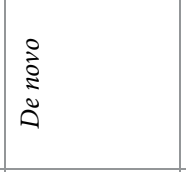 & 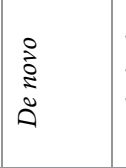 & 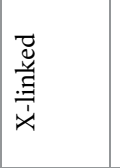 & 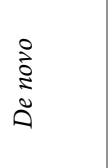 \\
\hline 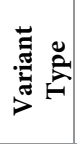 & 莺 & 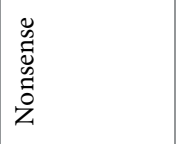 & 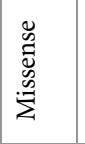 & 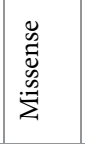 & 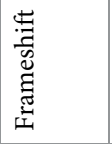 & 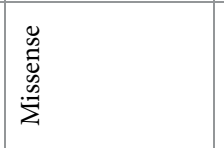 & 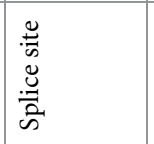 & 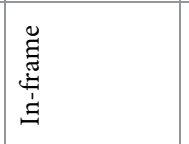 & 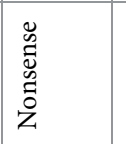 & 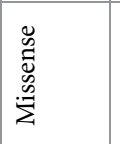 & 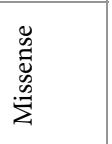 \\
\hline 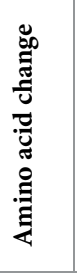 & 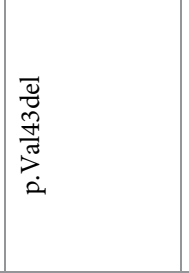 & 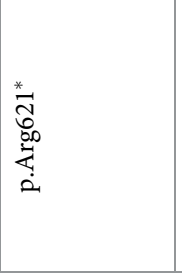 & 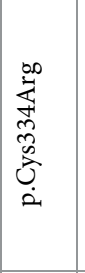 & 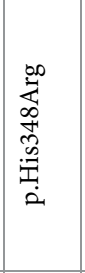 & 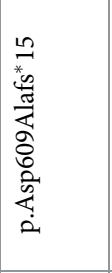 & 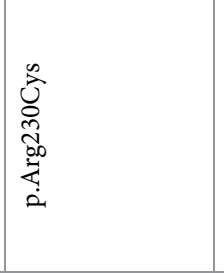 & 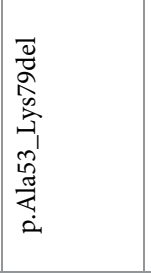 & 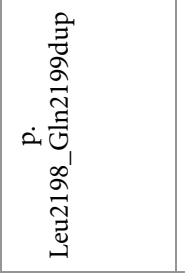 & 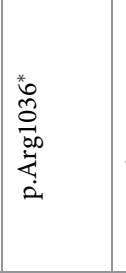 & 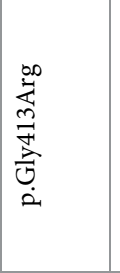 & 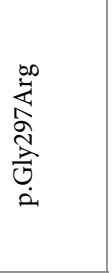 \\
\hline 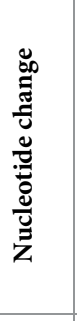 & 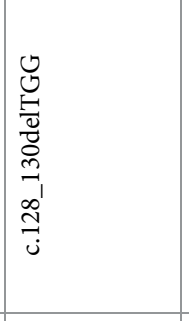 & 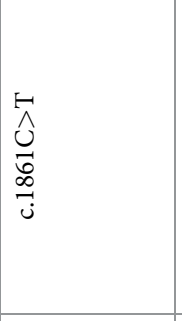 & \begin{tabular}{l}
$u$ \\
$\hat{\Lambda}$ \\
$\hat{O}$ \\
$o$ \\
\hdashline \\
$ن$
\end{tabular} & 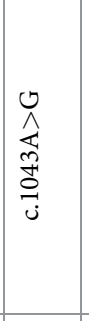 & 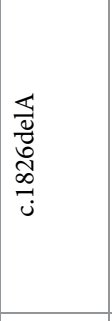 & $\begin{array}{l}\hat{N} \\
0 \\
\infty \\
0 \\
0 \\
ن\end{array}$ & $\begin{array}{l}\vec{A} \\
\hat{O} \\
+ \\
\hat{+} \\
\hat{N} \\
\mathrm{~J}\end{array}$ & 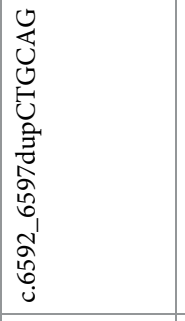 & 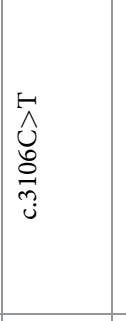 & 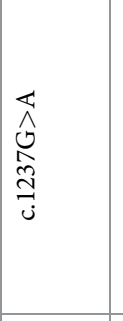 & $\begin{array}{l}\overleftrightarrow{\hat{~}} \\
\hat{0} \\
\infty \\
\infty \\
0\end{array}$ \\
\hline 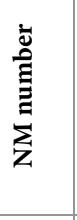 & 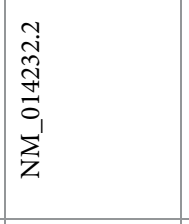 & 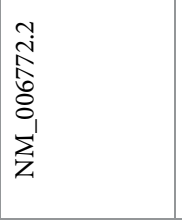 & 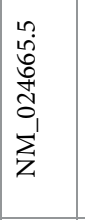 & 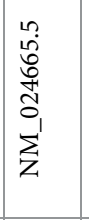 & 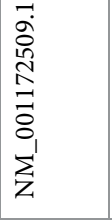 & 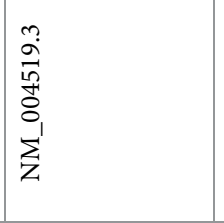 & 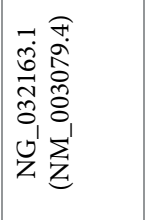 & 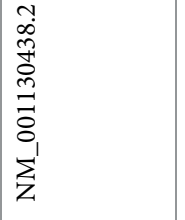 & 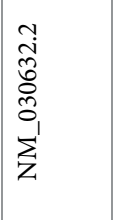 & 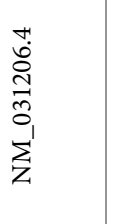 & 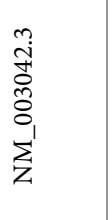 \\
\hline ভँّ & $\sum_{5}^{\nwarrow}$ & 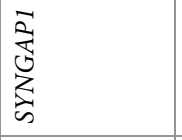 & $\underset{\sharp}{\stackrel{a}{\sharp}}$ & 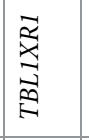 & 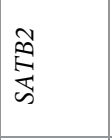 & 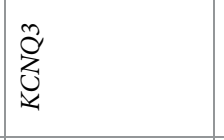 & 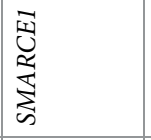 & 交 & $\begin{array}{l}3 \\
\hat{Z} \\
\hat{\psi}\end{array}$ & $\begin{array}{l}\overrightarrow{3} \\
\mathbb{5}\end{array}$ & 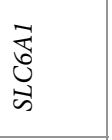 \\
\hline 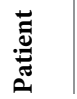 & - & $N$ & $m$ & $H$ & in & 6 & $n$ & $\infty$ & $a$ & $\stackrel{ }{ }$ & $\Xi$ \\
\hline
\end{tabular}


Table 2. Characteristics of AS-like patients at clinical re-evaluation.

\begin{tabular}{|c|c|c|c|c|c|c|c|c|c|c|c|}
\hline \multirow[t]{2}{*}{ Patient } & \multirow[t]{2}{*}{ Gender } & \multirow{2}{*}{$\begin{array}{c}\text { Age at } \\
\text { molecular } \\
\text { diagnosis } \\
\text { (years) }\end{array}$} & \multicolumn{4}{|c|}{ Consistent features present in $100 \%$ of patients with AS } & \multicolumn{3}{|c|}{$\begin{array}{l}\text { Frequent features present in more } \\
\text { than } 80 \% \text { of AS affected individuals }\end{array}$} & \multirow{2}{*}{\begin{tabular}{|c|} 
Associated \\
features \\
present in 20- \\
$\mathbf{8 0 \%}$ of AS \\
affected \\
individuals
\end{tabular}} & \multirow[t]{2}{*}{$\begin{array}{c}\text { Additional } \\
\text { clinical features }\end{array}$} \\
\hline & & & \begin{tabular}{|c|} 
Severe \\
developmental \\
delay
\end{tabular} & $\begin{array}{l}\text { Speech } \\
\text { impairment }\end{array}$ & $\begin{array}{c}\text { Ataxia or } \\
\text { unsteady } \\
\text { gait }\end{array}$ & $\begin{array}{c}\text { Apparent } \\
\text { happy } \\
\text { demeanor/ } \\
\text { Stereotypies }\end{array}$ & Microcephaly & Seizures & $\begin{array}{l}\text { Abnormal } \\
\text { EEG }\end{array}$ & & \\
\hline 1 & M & 14 & + & $\begin{array}{l}+(5-10 \\
\text { words })\end{array}$ & + & $+/+$ & - & + & + & $\begin{array}{c}\text { Sleep } \\
\text { disorder, } \\
\text { hypotonia }\end{array}$ & $\begin{array}{l}\text { Congenital } \\
\text { torticolis, } \\
\text { bruxism, } \\
\text { aggressive } \\
\text { behavior }\end{array}$ \\
\hline 2 & $\mathrm{~F}$ & 19 & + & $\begin{array}{l}+ \text { (less than } \\
5 \text { words) }\end{array}$ & - & $+/+$ & - & + & + & \begin{tabular}{|c|} 
Sleep \\
disorder, \\
feeding \\
problems, \\
kyphoscoliosis
\end{tabular} & $\begin{array}{l}\text { Aggressive } \\
\text { behavior }\end{array}$ \\
\hline 3 & $\mathrm{~F}$ & 12 & + & $\begin{array}{c}+(\text { Absent } \\
\text { speech })\end{array}$ & + & $-1+$ & - & + & + & Hypotonia & $\begin{array}{l}\text { Aggressive } \\
\text { behavior }\end{array}$ \\
\hline 4 & $\mathrm{~F}$ & 9 & + & $\begin{array}{l}+(\text { Absent } \\
\text { speech })\end{array}$ & + & $+/+$ & $+($ Relative $)$ & - & + & $\begin{array}{l}\text { Hypotonia, } \\
\text { feeding } \\
\text { problems }\end{array}$ & - \\
\hline 5 & $\mathrm{~F}$ & 20 & + & $\begin{array}{l}+(5-10 \\
\text { words })\end{array}$ & + & $+/-$ & - & - & + & - & $\begin{array}{c}\text { Dental anomalies, } \\
\text { auto and hetero- } \\
\text { aggressive } \\
\text { behavior }\end{array}$ \\
\hline 6 & $\mathrm{~F}$ & 18 & + & $\begin{array}{c}+ \text { (Absent } \\
\text { speech) }\end{array}$ & - & $+/-$ & + (Relative) & + & - & Scoliosis & - \\
\hline 7 & M & 15 & + & $\begin{array}{l}+ \text { (less than } \\
5 \text { words) }\end{array}$ & + & $+/+$ & - & + & + & $\begin{array}{l}\text { Feeding } \\
\text { problems, } \\
\text { wide mouth, } \\
\text { hypotonia }\end{array}$ & $\begin{array}{l}\text { Sparse scalp hair, } \\
\text { hypertricosis in } \\
\text { the back and } \\
\text { hypoplasia of the } \\
\text { corpus callosum, } \\
\text { hypoplasic } 5^{\text {th }} \\
\text { fingernail, auto } \\
\text { and hetero- } \\
\text { aggressive } \\
\text { behavior }\end{array}$ \\
\hline 8 & $\mathrm{~F}$ & 14 & + & $\begin{array}{l}+ \text { (More } \\
\text { than } 20 \\
\text { words) }\end{array}$ & + & $+/-$ & + & - & - & Hypotonia & $\begin{array}{l}\text { Cerebellar } \\
\text { atrophy }\end{array}$ \\
\hline 9 & M & 38 & + & $\begin{array}{l}+(\text { Absent } \\
\text { speech })\end{array}$ & + & $+/+$ & + (Relative) & + & + & $\begin{array}{l}\text { Hypotonia, } \\
\text { feeding } \\
\text { problems } \\
\text { (esophageal } \\
\text { reflux), sleep } \\
\text { disorder }\end{array}$ & $\begin{array}{l}\text { Dental anomalies, } \\
\text { bruxism, episodic } \\
\text { hyperventilation }\end{array}$ \\
\hline 10 & M & 7 & + & $\begin{array}{c}+ \text { (less than } \\
5 \text { words) }\end{array}$ & - & $+/-$ & + (Relative) & - & - & $\begin{array}{c}\text { Wide spaced } \\
\text { teeth, } \\
\text { brachycephaly }\end{array}$ & $\begin{array}{l}\text { Truncal obesity, } \\
\text { short stature }\end{array}$ \\
\hline 11 & $\mathrm{~F}$ & 14 & + & $\begin{array}{l}+ \text { (less than } \\
5 \text { words) }\end{array}$ & - & $+/+$ & + (Relative) & + & - & $\begin{array}{c}\text { Feeding } \\
\text { problems } \\
\text { (dysphagia) }\end{array}$ & - \\
\hline 12 & M & 24 & + & $\begin{array}{l}+(5-10 \\
\text { words })\end{array}$ & - & $+/+$ & + & + & NA & $\begin{array}{c}\text { Strabismus, } \\
\text { sleep disorder, } \\
\text { kyphoscoliosis }\end{array}$ & $\begin{array}{l}\text { Hypoplasia of the } \\
\text { corpus callosum, } \\
\text { abnormal } \\
\text { behavior, } \\
\text { hypothyroidism, } \\
\text { bruxism }\end{array}$ \\
\hline
\end{tabular}

(Continued) 
Table 2. (Continued)

\begin{tabular}{|c|c|c|c|c|c|c|c|c|c|c|c|}
\hline \multirow[t]{2}{*}{ Patient } & \multirow[t]{2}{*}{ Gender } & \multirow{2}{*}{$\begin{array}{c}\text { Age at } \\
\text { molecular } \\
\text { diagnosis } \\
\text { (years) }\end{array}$} & \multicolumn{4}{|c|}{ Consistent features present in $100 \%$ of patients with AS } & \multicolumn{3}{|c|}{$\begin{array}{l}\text { Frequent features present in more } \\
\text { than } 80 \% \text { of AS affected individuals }\end{array}$} & \multirow{2}{*}{\begin{tabular}{|c|} 
Associated \\
features \\
present in $20-$ \\
$\mathbf{8 0} \%$ of AS \\
affected \\
individuals
\end{tabular}} & \multirow[t]{2}{*}{$\begin{array}{c}\text { Additional } \\
\text { clinical features }\end{array}$} \\
\hline & & & $\begin{array}{c}\text { Severe } \\
\text { developmental } \\
\text { delay }\end{array}$ & $\begin{array}{c}\text { Speech } \\
\text { impairment }\end{array}$ & $\begin{array}{c}\text { Ataxia or } \\
\text { unsteady } \\
\text { gait }\end{array}$ & $\begin{array}{c}\text { Apparent } \\
\text { happy } \\
\text { demeanor/ } \\
\text { Stereotypies }\end{array}$ & Microcephaly & Seizures & $\begin{array}{l}\text { Abnormal } \\
\text { EEG }\end{array}$ & & \\
\hline 13 & M & 9 & + & $\begin{array}{c}+ \text { (Absent } \\
\text { speech) }\end{array}$ & + & $+/+$ & $+($ Relative) & + & - & $\begin{array}{c}\text { Sleep } \\
\text { disorder, } \\
\text { hypotonia }\end{array}$ & $\begin{array}{c}\text { Episodic } \\
\text { hyperventilation, } \\
\text { mild subcortical } \\
\text { atrophy }\end{array}$ \\
\hline 14 & M & 13 & + & $\begin{array}{c}+ \text { (Absent } \\
\text { speech })\end{array}$ & + & $+/+$ & - & + & + & $\begin{array}{c}\text { Sleep } \\
\text { disorder, } \\
\text { wide-spaced } \\
\text { teeth }\end{array}$ & $\begin{array}{l}\text { Myoclonic atonic } \\
\text { seizures, bruxism }\end{array}$ \\
\hline
\end{tabular}

M, Male; F, Female; +, present; -, not present; NA, non-available data.

the activation of the nonsense-mediated mRNA decay (NMD) machinery [51] and supporting a loss of function mechanism of disease for the HSF2 gene. Expression analysis of HSF2 in blood mRNA also showed a reduction in HSF2 expression in the patient compared to control (p-value 0.014) (S1B Fig). However, other tissues should be examined to clearly demonstrate the activation of NMD and a loss of function mechanism for the HSF2 variant.

Furthermore, HSF2 knockout mice show defects in the development of the central nervous system and spermatogenesis $[52,53]$. The identification of additional patients with loss of function variants in HSF 2 and functional studies in neural cells will contribute to elucidate the role of HSF2 in the AS-like phenotype.

De novo variants have been described to account for approximately half of the genetic architecture of severe developmental disorders [54]. In our cohort, 10 of the 11 pathogenic and likely pathogenic variants were de novo, accounting for $90 \%$ of diagnosis and highlighting the power of using trio-WES for the molecular diagnosis of severe developmental disorders. Only in one case, the X-linked variant in $L A S 1 L$ was inherited from the mother, who was a healthy carrier (data not shown).

All patients had received a suspected clinical diagnosis of AS. In the majority of our patients (12/14) the initial diagnosis was done during infancy or early childhood (before five years old). At the time of initial diagnosis, all of them presented severe global DD and speech impairment in addition to the characteristic happy disposition. Clinical re-evaluation at the time of molecular diagnosis confirmed the clinical diagnosis of AS (Table 2). In addition, other clinical features manifested during growth were then associated with the new identified genes. Pathogenic/likely pathogenic variants in VAMP2, KCNQ3, SMARCE1, SATB2, SYNGAP1, SLC6A1, ASXL3, SPTAN1, TBL1XR1 and LAS1L genes are associated with neurodevelopment disorders that overlap with AS and whose features have been defined in the last years [6,1319,21]. Finally, not all patients presented all the clinical features associated with the genes identified. This clinical variability, possibly due to the different pathogenicity strength of the genetic variants, differences in genetic background and to non-genetic environmental factors, makes the clinical diagnosis challenging.

Lack of molecular diagnosis in 10-15\% of clinically diagnosed AS patients has been used to define the AS-like group. Our trio based WES approach demonstrates that the majority of these patients $(78,5 \%)$ are carriers of pathogenic variants in genes involved in neurodevelopmental disorders whose features overlap with AS (Fig 1), highlighting the wide genetic 


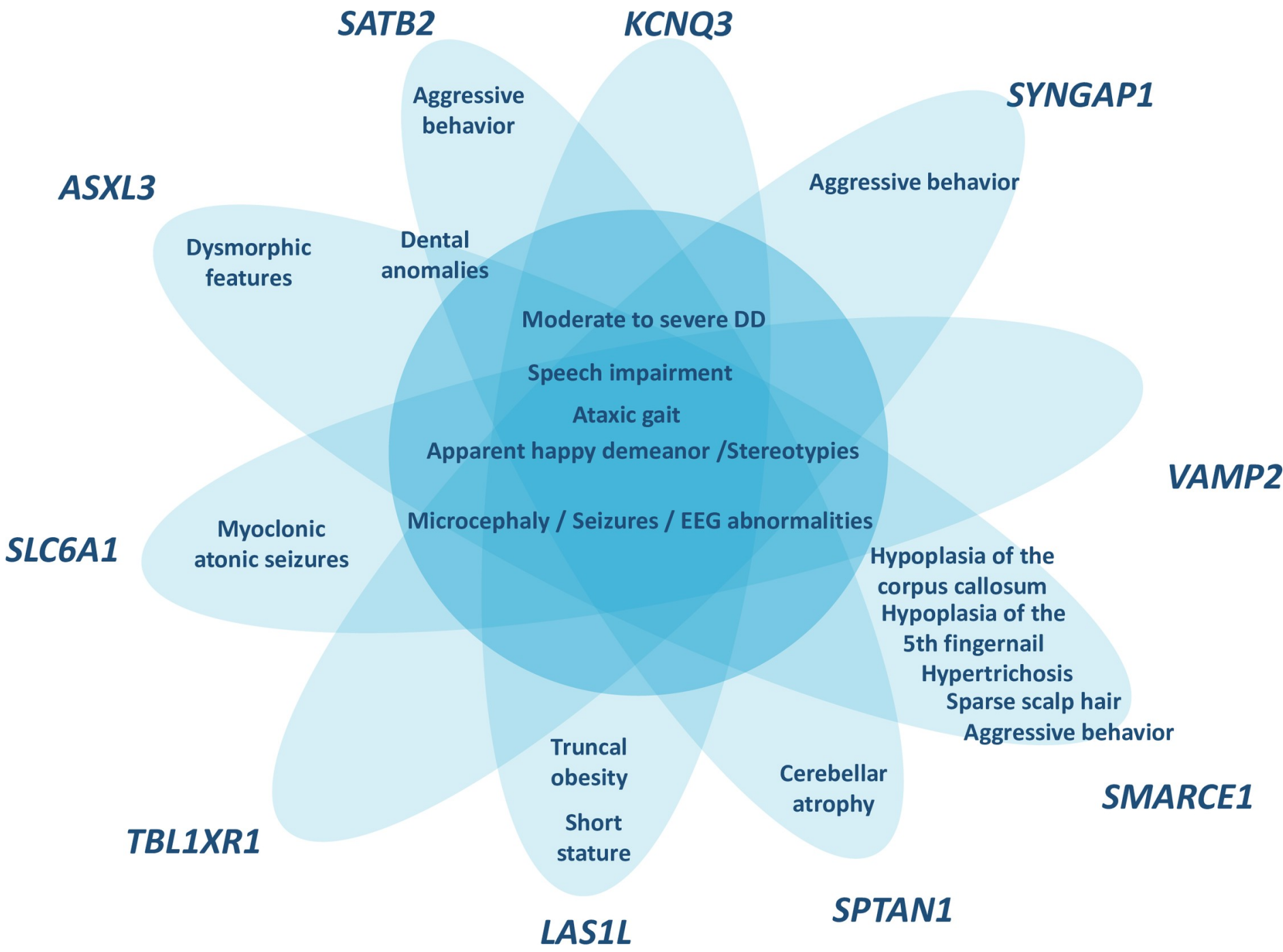

Fig 1. Schematic representation of the phenotypic overlap between the patients with pathogenic/likely pathogenic variants genes and the AS phenotype. In the middle of the figure the core AS features present in all the patients while in the tips the clinical features present in the patients that are associated with the gene identified.

https://doi.org/10.1371/journal.pone.0258766.g001

heterogeneity in AS-like patients and expanding the differential diagnosis. Likewise, other studies have recently described new genes associated with Angelman-like phenotypes such as HIVEP2 [55] or UNC80 [56].

TBL1XR1, SATB2, SMARCE1 and ASXL3 are known transcriptional regulators acting through chromatin modification $[46,57,58]$. UBE3A has been shown to be present in euchromatin-rich nuclear domains indicating that it may influence neuronal physiology by regulating chromatin and gene transcription [59]. RNA-seq studies of UBE3A loss in rat cortex, mice hippocampus and SH-SY5Y cells have shown differential gene expression of KCNQ3 [60], SMARCE1, HSF2 [38], SPTAN1 and SATB2 [39] suggesting that these genes may be transcriptionally regulated by UBE3A. Moreover, UBE3A gain and loss in human SH-SY5Y cells has been shown to have significant effects on DNA methylation and chromatin modification in genes involved in transcriptional regulation and brain development including SATB2, ASXL3, SMARCE1 and TBL1XR1 [38]. Overall, these evidences suggest common deregulated pathways between new identified Angelman-like genes and UBE3A. In addition, UBE3A has been 
shown to localize in axon terminals suggesting it locally regulates individual synapses. Five of the genes identified here, VAMP2, SYNGAP1, SLC6A1 and KCNQ3 are known to be involved in synapse function suggesting they may be regulated by UBE3A, as has already been demonstrated for KCNQ3 [60].

Except for the SYNGAP1 gene, none of the genes identified here have been previously described in the differential diagnosis of AS [61]. We propose the genes identified in this study should be included in the AS differential diagnosis and that trio WES should be considered as first line approach for the molecular diagnosis of AS-like patients. A high rate of diagnosis is essential since it contributes to more appropriate clinical patient surveillance as well as family genetic counseling.

\section{Supporting information}

S1 Fig. Quantification of $H S F 2$ mRNA transcripts suggest that variant c.456_459delTGAG is sensitive to nonsense-mediated mRNA decay (NMD). A) Sanger sequencing of a fragment encompassing variant c.456_459delTGAG from patient 12 and a control sample shows a reduction in the percentage of the allele with the variant in the cDNA compared to DNA. The sequence corresponds to the reverse strand. B) qPCR analysis of HSF2 gene expression in patient 12 and a control sample normalized to GAPDH shows less HSF2 expression in patient $12\left({ }^{*}\right.$ p-value 0.014$)$.

S1 Table. Missense, in-frame and splice site variant predictors. Prob. DA, Probably Damaging; Pos. DA, Possibly Damaging; D, Deleterious; M, Medium; H, High; NA, Not Available. (TIF)

\section{Acknowledgments}

We thank the patients and families for their participation in this study.

\section{Author Contributions}

Conceptualization: Elisabeth Gabau, Núria Capdevila, Neus Baena, Miriam Guitart, Anna Ruiz.

Data curation: Cinthia Aguilera, Ariadna Ramirez-Mallafré, Carme Brun-Gasca, Jana Dominguez-Carral, Veronica Delgadillo, Sophia Derdak, Nino Spataro, Neus Baena, Miriam Guitart, Anna Ruiz.

Formal analysis: Cinthia Aguilera, Elisabeth Gabau, Ariadna Ramirez-Mallafré, Carme BrunGasca, Jana Dominguez-Carral, Veronica Delgadillo, Steve Laurie, Sophia Derdak, Natàlia Padilla, Xavier de la Cruz, Núria Capdevila, Nino Spataro, Neus Baena, Miriam Guitart, Anna Ruiz.

Funding acquisition: Cinthia Aguilera, Elisabeth Gabau, Neus Baena, Miriam Guitart, Anna Ruiz.

Investigation: Cinthia Aguilera, Miriam Guitart, Anna Ruiz.

Methodology: Cinthia Aguilera, Elisabeth Gabau, Ariadna Ramirez-Mallafré, Carme BrunGasca, Natàlia Padilla, Xavier de la Cruz, Nino Spataro, Miriam Guitart, Anna Ruiz.

Project administration: Miriam Guitart.

Resources: Elisabeth Gabau, Núria Capdevila. 
Software: Steve Laurie, Sophia Derdak, Natàlia Padilla, Xavier de la Cruz, Nino Spataro.

Supervision: Miriam Guitart, Anna Ruiz.

Validation: Cinthia Aguilera, Nino Spataro, Miriam Guitart, Anna Ruiz.

Visualization: Cinthia Aguilera.

Writing - original draft: Cinthia Aguilera, Anna Ruiz.

Writing - review \& editing: Cinthia Aguilera, Elisabeth Gabau, Ariadna Ramirez-Mallafré,

Carme Brun-Gasca, Jana Dominguez-Carral, Veronica Delgadillo, Steve Laurie, Sophia

Derdak, Natàlia Padilla, Xavier de la Cruz, Núria Capdevila, Nino Spataro, Neus Baena,

Miriam Guitart, Anna Ruiz.

\section{References}

1. Williams $\mathrm{C}$, Beaudet $\mathrm{AL}$, Clayton-Smith J, Knoll JH, Kyllerman M, Laan LA, et al. Angelman Syndrome 2005: Updated Consensus for Diagnostic Criteria Charles. Am J Med Genet Part A. 2006; 140: 413418. https://doi.org/10.1002/ajmg.a.31074 PMID: 16470747

2. Beygo J, Buiting K, Ramsden SC, Ellis R, Clayton-Smith J, Kanber D. Update of the EMQN/ACGS best practice guidelines for molecular analysis of Prader-Willi and Angelman syndromes. Eur J Hum Genet. 2019; 27: 1326-1340. https://doi.org/10.1038/s41431-019-0435-0 PMID: 31235867

3. Tan WH, Bird LM, Thibert RL, Williams CA. If not Angelman, what is it? A review of Angelman-like syndromes. Am J Med Genet Part A. 2014; 164: 975-992. https://doi.org/10.1002/ajmg.a.36416 PMID: 24779060

4. Luk H-M. Angelman-Like Syndrome: A Genetic Approach to Diagnosis with Illustrative Cases. Case Rep Genet. 2016; 2016: 1-6. https://doi.org/10.1155/2016/9790169 PMID: 26942024

5. Harlalka G V, Baple EL, Cross H, Kuhnle S, Cubillos-Rojas M, Matentzoglu K, et al. Mutation of HERC2 causes developmental delay with Angelman-like features. J Med Genet. 2013; 50: 65-73. https://doi. org/10.1136/jmedgenet-2012-101367 PMID: 23243086

6. Salpietro V, Malintan NT, Llano-Rivas I, Spaeth CG, Efthymiou S, Striano P, et al. Mutations in the Neuronal Vesicular SNARE VAMP2 Affect Synaptic Membrane Fusion and Impair Human Neurodevelopment. Am J Hum Genet. 2019; 104: 721-730. https://doi.org/10.1016/j.ajhg.2019.02.016 PMID: 30929742

7. $\mathrm{Li} \mathrm{H}$, Durbin R. Fast and accurate long-read alignment with Burrows-Wheeler transform. Bioinformatics. 2010; 26: 589-595. https://doi.org/10.1093/bioinformatics/btp698 PMID: 20080505

8. Van der Auwera GA, Carneiro MO, Hartl C, Poplin R, Del Angel G, Levy-Moonshine A, et al. From Fast $Q$ data to high confidence variant calls: the Genome Analysis Toolkit best practices pipeline. Curr Protoc Bioinforma. 2013; 43: 11.10.1-33. https://doi.org/10.1002/0471250953.bi1110s43 PMID: 25431634

9. Wang $\mathrm{K}, \mathrm{Li} \mathrm{M}$, Hakonarson $\mathrm{H}$. ANNOVAR: functional annotation of genetic variants from high-throughput sequencing data. Nucleic Acids Res. 2010; 38: e164. https://doi.org/10.1093/nar/gkq603 PMID: 20601685

10. Desmet F-O, Hamroun D, Lalande M, Collod-Beroud G, Claustres M, Beroud C. Human Splicing Finder: an online bioinformatics tool to predict splicing signals. Nucleic Acids Res. 2009; 37: e67. https://doi.org/10.1093/nar/gkp215 PMID: 19339519

11. Richards S, Aziz N, Bale S, Bick D, Das S, Gastier-Foster J, et al. Standards and guidelines for the interpretation of sequence variants: a joint consensus recommendation of the American College of Medical Genetics and Genomics and the Association for Molecular Pathology. Genet Med. 2015; 17: 405-423. https://doi.org/10.1038/gim.2015.30 PMID: 25741868

12. Landrum MJ, Lee JM, Benson M, Brown GR, Chao C, Chitipiralla S, et al. ClinVar: improving access to variant interpretations and supporting evidence. Nucleic Acids Res. 2018; 46: D1062-D1067. https:// doi.org/10.1093/nar/gkx1153 PMID: 29165669

13. Syrbe S, Harms FL, Parrini E, Montomoli M, Mutze U, Helbig KL, et al. Delineating SPTAN1 associated phenotypes: from isolated epilepsy to encephalopathy with progressive brain atrophy. Brain. 2017; 140: 2322-2336. https://doi.org/10.1093/brain/awx195 PMID: 29050398

14. Balasubramanian M, Willoughby J, Fry AE, Weber A, Firth H V, Deshpande C, et al. Delineating the phenotypic spectrum of Bainbridge-Ropers syndrome: 12 new patients with de novo, heterozygous, 
loss-of-function mutations in ASXL3 and review of published literature. J Med Genet. 2017; 54: 537543. https://doi.org/10.1136/jmedgenet-2016-104360 PMID: 28100473

15. Johannesen KM, Gardella E, Linnankivi T, Courage C, Martin A de Saint, Lehesjoki A-E, et al. Defining the phenotypic spectrum of SLC6A1 mutations. Epilepsia. 2018; 59: 389-402. https://doi.org/10.1111/ epi.13986 PMID: 29315614

16. Hu H, Haas S, Chelly J, Esch H Van, Raynaud M, Brouwer A de, et al. X-exome sequencing of 405 unresolved families identifies seven novel intellectual disability genes. Mol Psychiatry. 2015; 21: 133148. https://doi.org/10.1038/mp.2014.193 PMID: 25644381

17. Mignot C, von Stulpnagel C, Nava C, Ville D, Sanlaville D, Lesca G, et al. Genetic and neurodevelopmental spectrum of SYNGAP1-associated intellectual disability and epilepsy. J Med Genet. 2016; 53: 511-522. https://doi.org/10.1136/jmedgenet-2015-103451 PMID: 26989088

18. Laskowski RA, Thornton JM, Tyagi N, Johnson D, McWilliam C, Kinning E, et al. Integrating population variation and protein structural analysis to improve clinical interpretation of missense variation: application to the WD40 domain. Hum Mol Genet. 2016; 25: 927-935. https://doi.org/10.1093/hmg/ddv625 PMID: 26740553

19. Zarate YA, Smith-Hicks CL, Greene C, Abbott MA, Siu VM, Calhoun ARUL, et al. Natural history and genotype-phenotype correlations in 72 individuals with SATB2-associated syndrome. Am J Med Genet Part A. 2018; 176: 925-935. https://doi.org/10.1002/ajmg.a.38630 PMID: 29436146

20. Sands TT, Miceli F, Lesca G, Beck AE, Sadleir LG, Arrington DK, et al. Autism and developmental disability caused by KCNQ3 gain-of-function variants. Ann Neurol. 2019. https://doi.org/10.1002/ana. 25522 PMID: 31177578

21. Zarate YA, Bhoj E, Kaylor J, Li D, Tsurusaki Y, Miyake N, et al. SMARCE1, a rare cause of Coffin-Siris Syndrome: Clinical description of three additional cases. Am J Med Genet Part A. 2016; 170: 19671973. https://doi.org/10.1002/ajmg.a.37722 PMID: 27264197

22. Firth H V, Richards SM, Bevan AP, Clayton S, Corpas M, Rajan D, et al. DECIPHER: Database of Chromosomal Imbalance and Phenotype in Humans Using Ensembl Resources. Am J Hum Genet. 2009; 84: 524-533. https://doi.org/10.1016/j.ajhg.2009.03.010 PMID: 19344873

23. Miceli F, Soldovieri MV, Ambrosino P, Maria M De, Migliore M, Migliore Xr, et al. Early-Onset Epileptic Encephalopathy Caused by Gain-of-Function Mutations in the Voltage Sensor of Kv7.2 and Kv7.3 Potassium Channel Subunits. J Neurosci. 2015; 35: 3782-3793. https://doi.org/10.1523/JNEUROSCI. 4423-14.2015 PMID: 25740509

24. Aguilera C, Gabau E, Laurie S, Baena N, Derdak S, Capdevila N, et al. Identification of a de novo splicing variant in the Coffin-Siris gene, SMARCE1, in a patient with Angelman-like syndrome. Mol Genet genomic Med. 2019; 7: e00511. https://doi.org/10.1002/mgg3.511 PMID: 30548424

25. Kuechler A, Czeschik JC, Graf E, Grasshoff U, Huffmeier U, Busa T, et al. Bainbridge-Ropers syndrome caused by loss-of-function variants in ASXL3: a recognizable condition. Eur J Hum Genet. 2017; 25: 183-191. https://doi.org/10.1038/ejhg.2016.165 PMID: 27901041

26. Carvill GL, McMahon JM, Schneider A, Zemel M, Myers CT, Saykally J, et al. Mutations in the GABA Transporter SLC6A1 Cause Epilepsy with Myoclonic-Atonic Seizures. Am J Hum Genet. 2015; 96: 808-815. https://doi.org/10.1016/j.ajhg.2015.02.016 PMID: 25865495

27. Bell L, Wittkowski A, Hare DJ. Movement Disorders and Syndromic Autism: A Systematic Review. J Autism Dev Disord. 2019; 49: 54-67. https://doi.org/10.1007/s10803-018-3658-y PMID: 30014250

28. Huang L, Kinnucan E, Wang G, Beaudenon S, Howley PM, Huibregtse JM, et al. Structure of an E6APUbcH7 complex: insights into ubiquitination by the E2-E3 enzyme cascade. Science. 1999; 286: 13211326. https://doi.org/10.1126/science.286.5443.1321 PMID: 10558980

29. Martinez-Noel G, Luck K, Kuhnle S, Desbuleux A, Szajner P, Galligan JT, et al. Network Analysis of UBE3A/E6AP-Associated Proteins Provides Connections to Several Distinct Cellular Processes. J Mol Biol. 2018; 430: 1024-1050. https://doi.org/10.1016/j.jmb.2018.01.021 PMID: 29426014

30. Kléber M, Sommer L. Wnt signaling and the regulation of stem cell function. Curr Opin Cell Biol. 2004; 16: 681-687. https://doi.org/10.1016/j.ceb.2004.08.006 PMID: 15530781

31. Lie D-C, Colamarino SA, Song H-J, Désiré L, Mira H, Consiglio A, et al. Wnt signalling regulates adult hippocampal neurogenesis. Nature. 2005; 437: 1370-1375. https://doi.org/10.1038/nature04108 PMID: 16251967

32. Kuwabara T, Hsieh J, Muotri A, Yeo G, Warashina M, Lie DC, et al. Wnt-mediated activation of NeuroD1 and retro-elements during adult neurogenesis. Nat Neurosci. 2009; 12: 1097-1105. https://doi.org/10. 1038/nn.2360 PMID: 19701198

33. Sun AX, Yuan Q, Tan S, Xiao Y, Wang D, Khoo ATT, et al. Direct Induction and Functional Maturation of Forebrain GABAergic Neurons from Human Pluripotent Stem Cells. Cell Rep. 2016; 16: 1942-1953. https://doi.org/10.1016/j.celrep.2016.07.035 PMID: 27498872 
34. Wallace ML, Burette AC, Weinberg RJ, Philpot BD. Maternal Loss of Ube3a Produces an Excitatory/ Inhibitory Imbalance through Neuron Type-Specific Synaptic Defects. Neuron. 2012; 74: 793-800. https://doi.org/10.1016/j.neuron.2012.03.036 PMID: 22681684

35. Margolis SS, Salogiannis J, Lipton DM, Mandel-Brehm C, Wills ZP, Mardinly AR, et al. EphB-Mediated Degradation of the RhoA GEF Ephexin5 Relieves a Developmental Brake on Excitatory Synapse Formation. Cell. 2010; 143: 442-455. https://doi.org/10.1016/j.cell.2010.09.038 PMID: 21029865

36. Kühnle $S$, Mothes $B$, Matentzoglu K, Scheffner M. Role of the ubiquitin ligase E6AP/UBE3A in controlling levels of the synaptic protein Arc. Proc Natl Acad Sci U S A. 2013; 110: 8888-8893. https://doi.org/ 10.1073/pnas.1302792110 PMID: 23671107

37. Zaaroor-Regev D, de Bie P, Scheffner M, Noy T, Shemer R, Heled M, et al. Regulation of the polycomb protein Ring1B by self-ubiquitination or by E6-AP may have implications to the pathogenesis of Angelman syndrome. Proc Natl Acad Sci U S A. 2010; 107: 6788-6793. https://doi.org/10.1073/pnas. 1003108107 PMID: 20351251

38. Lopez SJ, Dunaway K, Islam MS, Mordaunt C, Vogel Ciernia A, Meguro-Horike M, et al. UBE3A-mediated regulation of imprinted genes and epigenome-wide marks in human neurons. Epigenetics. 2017/ 11/06. 2017; 12: 982-990. https://doi.org/10.1080/15592294.2017.1376151 PMID: 28925810

39. Lopez SJ, Laufer BI, Beitnere U, Berg EL, Silverman JL, O'Geen H, et al. Imprinting effects of UBE3A loss on synaptic gene networks and Wnt signaling pathways. Hum Mol Genet. 2019; 28: 3842-3852. https://doi.org/10.1093/hmg/ddz221 PMID: 31625566

40. El Hokayem J, Weeber E, Nawaz Z. Loss of Angelman Syndrome Protein E6AP Disrupts a Novel Antagonistic Estrogen-Retinoic Acid Transcriptional Crosstalk in Neurons. Mol Neurobiol. 2018; 55: 7187-7200. https://doi.org/10.1007/s12035-018-0871-9 PMID: 29388081

41. Südhof TC, Rothman JE. Membrane fusion: grappling with SNARE and SM proteins. Science. 2009; 323: 474-477. https://doi.org/10.1126/science.1161748 PMID: 19164740

42. Clement JP, Aceti M, Creson TK, Ozkan ED, Shi Y, Reish NJ, et al. Pathogenic SYNGAP1 mutations impair cognitive development by disrupting maturation of dendritic spine synapses. Cell. 2012; 151: 709-723. https://doi.org/10.1016/j.cell.2012.08.045 PMID: 23141534

43. Mermer F, Poliquin S, Rigsby K, Rastogi A, Shen W, Romero-Morales A, et al. Common molecular mechanisms of SLC6A1 variant-mediated neurodevelopmental disorders in astrocytes and neurons. Brain. 2021; 144: 2499-2512. https://doi.org/10.1093/brain/awab207 PMID: 34028503

44. Baculis BC, Zhang J, Chung HJ. The Role of Kv7 Channels in Neural Plasticity and Behavior. Front Physiol. 2020; 11: 1206. https://doi.org/10.3389/fphys.2020.568667 PMID: 33071824

45. Li JY, Daniels G, Wang J, Zhang X. TBL1XR1 in physiological and pathological states. Am J Clin Exp Urol. 2015; 3: 13-23. PMID: 26069883

46. Gyorgy AB, Szemes M, de Juan Romero C, Tarabykin V, Agoston D V. SATB2 interacts with chromatin-remodeling molecules in differentiating cortical neurons. Eur J Neurosci. 2008; 27: 865-873. https:// doi.org/10.1111/j.1460-9568.2008.06061.x PMID: 18333962

47. Lomeli $\mathrm{H}$, Castillo-Robles J. The developmental and pathogenic roles of BAF57, a special subunit of the BAF chromatin-remodeling complex. FEBS Lett. 2016; 590: 1555-1569. https://doi.org/10.1002/18733468.12201 PMID: 27149204

48. Functional Katoh M. and cancer genomics of ASXL family members. Br J Cancer. 2013; 109: 299-306. https://doi.org/10.1038/bjc.2013.281 PMID: 23736028

49. Clark MM, Stark Z, Farnaes L, Tan TY, White SM, Dimmock D, et al. Meta-analysis of the diagnostic and clinical utility of genome and exome sequencing and chromosomal microarray in children with suspected genetic diseases. npj Genomic Med. 2018; 3: 1-10. https://doi.org/10.1038/s41525-018-0053-8 PMID: 30002876

50. Consortium GTEx. The GTEx Consortium atlas of genetic regulatory effects across human tissues. Science (80-). 2020; 369: 1318 LP-1330. https://doi.org/10.1126/science.aaz1776 PMID: 32913098

51. Lykke-Andersen $\mathrm{S}$, Jensen $\mathrm{TH}$. Nonsense-mediated mRNA decay: an intricate machinery that shapes transcriptomes. Nat Rev Mol Cell Biol. 2015; 16: 665-677. https://doi.org/10.1038/nrm4063 PMID: 26397022

52. Wang G, Zhang J, Moskophidis D, Mivechi NF. Targeted disruption of the heat shock transcription factor (hsf)-2 gene results in increased embryonic lethality, neuronal defects, and reduced spermatogenesis. Genesis. 2003; 36: 48-61. https://doi.org/10.1002/gene.10200 PMID: 12748967

53. Kallio M, Chang Y, Manuel M, Alastalo T-P, Rallu M, Gitton Y, et al. Brain abnormalities, defective meiotic chromosome synapsis and female subfertility in HSF2 null mice. EMBO J. 2002; 21: 2591-2601. https://doi.org/10.1093/emboj/21.11.2591 PMID: 12032072 
54. McRae J, Clayton S, Fitzgerald T, Kaplanis J, Prigmore E, Rajan D, et al. Prevalence and architecture of de novo mutations in developmental disorders. Nature. 2017; 542: 433-438. https://doi.org/10.1038/ nature21062 PMID: 28135719

55. Goldsmith H, Wells A, Sá MJN, Williams M, Heussler H, Buckman M, et al. Expanding the phenotype of intellectual disability caused by HIVEP2 variants. Am J Med Genet A. 2019; 179: 1872-1877. https:// doi.org/10.1002/ajmg.a.61271 PMID: 31207095

56. He Y, Ji X, Yan H, Ye X, Liu Y, Wei W, et al. Biallelic UNC80 mutations caused infantile hypotonia with psychomotor retardation and characteristic facies 2 in two Chinese patients with variable phenotypes. Gene. 2018; 660: 13-17. https://doi.org/10.1016/j.gene.2018.03.063 PMID: 29572195

57. Srivastava A, Ritesh KC, Tsan Y-C, Liao R, Su F, Cao X, et al. De novo dominant ASXL3 mutations alter H2A deubiquitination and transcription in Bainbridge-Ropers syndrome. Hum Mol Genet. 2016; 25 597-608. https://doi.org/10.1093/hmg/ddv499 PMID: 26647312

58. Yoon H-G, Choi Y, Cole PA, Wong J. Reading and function of a histone code involved in targeting corepressor complexes for repression. Mol Cell Biol. 2005; 25: 324-335. https://doi.org/10.1128/MCB.25.1. 324-335.2005 PMID: 15601853

59. Burette AC, Judson MC, Burette S, Phend KD, Philpot BD, Weinberg RJ. Subcellular organization of UBE3A in neurons. J Comp Neurol. 2017; 525: 233-251. https://doi.org/10.1002/cne.24063 PMID: 27339004

60. Panov J, Kaphzan H. Bioinformatics analyses show dysregulation of calcium-related genes in Angelman syndrome mouse model. Neurobiol Dis. 2021; 148: 105180. https://doi.org/10.1016/j.nbd.2020. 105180 PMID: 33212289

61. Parker MJ, Magee AC, Maystadt I, Benoit V, study D, FitzPatrick DR, et al. De novo, heterozygous, loss-of-function mutations in SYNGAP1 cause a syndromic form of intellectual disability. Am J Med Genet Part A. 2015; 167: 2231-2237. https://doi.org/10.1002/ajmg.a.37189 PMID: 26079862 